\section{Estratificação de territórios prioritários para vigilância da doença de Chagas crônica: análise multicritério para tomada de decisão em saúde}

\author{
Stratification of priority territories for surveillance \\ of chronic Chagas disease: multicriteria analysis \\ for decision-making in health
}

\section{Estratificación de territorios prioritarios para la vigilancia de la enfermedad de Chagas crónica: análisis multicriterio para la toma de decisión en salud}

Mayara Maia Lima 1 Veruska Maia da Costa 1 Swamy Lima Palmeira 1 André Peres Barbosa de Castro 1

\title{
Resumo
}

Para o reconhecimento da magnitude da doença de Chagas crônica no Brasil, é necessário rearticular as ações de vigilância em saúde, buscando o desenvolvimento de uma ampla rede hierarquizada de serviços distribuida geograficamente, para prover atendimento aos milhares de cidadãos acometidos pela infecção por Trypanosoma cruzi. O objetivo do trabalho foi elaborar um modelo de priorização de municípios para a vigilância da doença de Chagas crônica, a fim de ofertar cuidado integral às pessoas afetadas pela enfermidade. Para isso, foi realizada uma análise multicritério utilizando o algoritmo PROMETHEÉ II implementado no software Pradin. Os critérios de avaliação do modelo foram compostos de três indices construídos a partir de indicadores (a) epidemiológicos diretamente relacionados à doença de Chagas crônica, (b) decorrentes da evolução da doença de Chagas crônica, e (c) relacionados ao acesso aos serviços de saúde. A Escala Fundamental de Saaty foi utilizada para definição dos pesos dos indicadores, com maior importância aos diretamente relacionados à doença de Chagas crônica e àqueles com maior confiabilidade e respectiva qualidade de informação. A avaliação da consistência dos modelos se deu em comparação com dados disponíveis das áreas historicamente endêmicas, com a distribuição de casos agudos, e outras análises de sensibilidade. O modelo mais adequado foi definido por 1.345 municípios de média prioridade, 1.003 de alta e 601 como muito alta prioridade para doença de Chagas crônica, com maiores proporções nas regiões Sudeste e Nordeste. A priorização permite à gestão racionalizar e direcionar recursos, sendo essencial para identificar os territórios onde as pessoas infectadas estão vivendo, a fim de promover a assistência integral e melhorar a qualidade de vida.

Doença de Chagas; Técnicas de Apoio para a Decisão;

Vigilância em Saúde Pública

Correspondência

M. M. Lima

Programa de Treinamento de Epidemiologia Aplicada aos Serviços do Sistema Único de Saúde, Secretaria de Vigilância em Saúde, Ministério da Saúde.

SRTVN, Quadra 701, Via W5 Norte, Lote D, Edifício PO700, 6o andar, Brasília, DF 70719-040, Brasil. may_maial@yahoo.com.br

1 Secretaria de Vigilância em Saúde, Ministério da Saúde, Brasília, Brasil. 


\section{Introdução}

A doença de Chagas é conhecida há mais de um século 1, e continua sendo um dos problemas de saúde pública mais prevalentes na América Latina 2,3. Cerca de 65 milhões de pessoas, que residem em 21 países endêmicos das Américas, estão sob risco, e 12 mil contraem a infecção anualmente 4. No Brasil, a doença é uma das condições parasitárias com maior carga de doença 5 .

A área com risco de transmissão vetorial (endêmica) no país, conhecida no final dos anos 1970, incluía 18 estados com mais de 2.200 municípios. A partir de 1975, o Ministério da Saúde iniciou ações de controle químico vetorial de forma sistematizada, que, junto à intensa vigilância de triagem de doadores de sangue e tecidos, reduziram o número de casos novos da doença de Chagas. Os resultados dessas ações foram demonstrados por Ostermayer et al. 6, por meio do inquérito nacional realizado entre os anos de 2001 e 2008, encontrando, em quase 105 mil crianças de até cinco anos de idade, provenientes de área rural, uma prevalência de 0,03\%. Porém, estima-se que a transmissão no século XX e início do século XXI tenha resultado em uma coorte de milhões de pessoas infectadas por Trypanosoma cruzi nas diferentes regiões 4.

A mudança do perfil epidemiológico, de um cenário de transmissão vetorial intradomiciliar para a transmissão ligada ao ciclo silvestre do parasito, concentrou os casos novos da doença na Região Amazônica, com cerca de 235 casos novos/ano 7 . Esse cenário demonstra a necessidade de rearticular as ações de vigilância em saúde, reforçando a importância de desenvolver um modelo amplo e integrador, para a tomada de decisão em diferentes esferas de gestão, que considere o reconhecimento de casos da infecção pelo protozoário.

Há limitações para reconhecimento e definição de áreas prioritárias para doença de Chagas, principalmente quanto à doença de Chagas crônica, cujo principal desafio é o conhecimento da distribuição territorial dos casos nessa fase, considerando que foram infectados há muito tempo e que as últimas décadas foram marcadas por intensa migração populacional 8 . Ressalta-se ainda a ausência de inquérito sorológico recente que permitisse conhecer a atual magnitude da doença na população.

Em 2020, a doença de Chagas crônica foi definida como uma doença de notificação compulsória nacionalmente 9 , e encontra-se em processo de estruturação a sistematização dos dados dos casos. Assim, permanece a limitação de fontes de dados atuais para construção de indicadores para doença de Chagas crônica. Nesse contexto, o presente estudo utilizou a ferramenta de apoio multicritério à decisão (AMD) para elaboração de um modelo de estratificação com o objetivo de eleger municípios prioritários para a vigilância em saúde da doença de Chagas crônica, de forma mais oportuna e eficiente no território, a fim de ofertar cuidado integral às pessoas afetadas pela forma crônica da doença.

\section{Métodos}

A AMD é um processo que utiliza um conjunto de abordagens qualitativas e quantitativas para tratar de situações nas quais se devem incorporar múltiplas considerações na tomada de decisão 10,11 e que permite a combinação de diferentes fontes de dados, reduzindo a limitação resultante de informações inexistentes ou sub-registros. Uma das técnicas do AMD para seleção ou ordenamento de alternativas é a denominada preference ranking method for enrichment evaluation (PROMETHEÉ) e suas variantes de I a VI. Este modelo é simples, tendo por objetivo final obter uma ordenação das alternativas de forma transparente e balanceada a partir dos critérios considerados relevantes para o problema em questão 12 .

Neste estudo, a AMD foi realizada por meio do algoritmo PROMETHEÉ II 13, utilizando o software Pradin 3.0 (http://www.anipes.org.br/).

Como fonte de dados, foram utilizados o Sistema de Informações sobre Mortalidade (SIM), Sistema de Internação Hospitalar (SIH), Sistema de Informações Ambulatoriais (SIA) e Sistema de Informação da Atenção Básica (SIAB) que possuíam registros de doença de Chagas por município de residência, nos anos de 2014 a 2016. Para os cálculos dos indicadores baseados na população, foram usadas as estimativas do Instituto Brasileiro de Geografia e Estatística (IBGE) para o Tribunal de Contas da União (TCU) e para população residente por faixa etária, foram empregadas as estimativas preliminares elaboradas pelo Ministério da Saúde. 


\section{Descrição dos indicadores}

Os critérios de avaliação das alternativas foram compostos por três índices: (a) Chagas - construído por indicadores epidemiológicos diretamente relacionados à doença de Chagas crônica; (b) Associadas - considerando doenças/agravos decorrentes da evolução da doença; (c) Acesso - com indicadores relacionados ao acesso aos serviços de saúde.

Os índices foram obtidos pela média aritmética dos indicadores (equação 1), os quais foram previamente normalizados para valores de 0 a 1. No Quadro 1, estão descritos os indicadores utilizados para a composição de cada índice (Acesso, Associadas e Chagas).

Valor do índice $=\left(\sum\right.$ (valor do indicador $x$ peso $\left.)\right) /($ número de indicadores do índice $)$

\section{Pesos dos indicadores e índices}

O método PROMETHEÉ II busca sistematizar a modelagem de preferências, ficando a definição dos pesos dos indicadores a critério do decisor. Para reduzir a subjetividade na definição dos pesos dos indicadores, uma vez que não é possível eliminá-la, e conferir maior transparência, utilizou-se a Escala Fundamental de Saaty, construída a partir de outro método de análise multicritério - análise hierárquica de processos (AHP) 14,15.

Com base na escala Saaty, conferiu-se maior importância para indicadores diretamente relacionados à doença de Chagas, com maior confiabilidade e respectiva qualidade da fonte de informação (p.ex.: SIM é mais confiável e tem menos limitações que SIAB). Para avaliar a consistência dos julgamentos na matriz de comparação, realizou-se o cálculo da razão de consistência (RC), padronizada por Saaty, sendo aceitável um valor menor que 0,10 .

\section{Simulações}

Considerando a complexidade de municípios brasileiros e de cenários para doença de Chagas, os municípios foram divididos em três grupos de comparação conforme porte populacional: grupo 1, municípios de pequeno porte (até 20.000 habitantes); grupo 2, pequeno porte II (20.001 a 50.000) e grupo 3, de médio, grande e metrópole (50.001 a 100.000, 100.001 a 900.000, superior a 900.000 habitantes, respectivamente).

Para cada grupo de porte populacional, foram feitas seis simulações no Pradin, para avaliar o efeito de mudanças nos pesos dos indicadores e índices, utilizando a função de preferência critério usual ou verdadeiro critério, visto que não há parâmetros definidos para os indicadores de doença de Chagas. Para o índice de acesso, na comparação entre os municípios, a lógica foi inversa aos indicadores epidemiológicos, com preferência estrita àqueles com menor valor.

Para a classificação dos municípios prioritários para doença de Chagas crônica no país, foi realizada quebra natural de Jenks 16 do indicador multicriterial (IMC) dos 5.570 municípios no programa QGIS 2.18 (https://www.qgis.org/), resultando em cinco grupos de prioridade: muito baixa; baixa; média; alta; muito alta. A classificação por quebras naturais aplica-se ao objetivo de priorização, pois permite agrupar valores similares e diferenciar melhor as classes definidas.

\section{Comparação entre as simulações e validação para tomada de decisão}

Para comparação entre simulações e validação para tomada de decisão, foi utilizado o diagrama de dispersão dos IMC entre as simulações e calculado o valor $\mathrm{R}^{2}$ para avaliar os efeitos das alterações. Arbitrou-se que $\mathrm{R}^{2}<0,5$ indica alteração relevante nos resultados; 0,5 $\leq \mathrm{R}^{2}<0,8$, impacto moderado; $\mathrm{R}^{2} \geq 0,8$, mudança irrelevante.

Considerando o deslocamento progressivo das pessoas infectadas por Tr. cruzi para idades mais avançadas e que pacientes crônicos identificados a partir dos 60 anos de idade provavelmente se infectaram a partir da década de 1950, foi realizada comparação com a lista de 729 municípios com ocorrência de Triatoma infestans no inquérito entomológico (1975-1983) 17, assim como no inquérito 
Quadro 1

Indicadores utilizados para a construção dos índices de Chagas, Associadas e Acesso.

\begin{tabular}{|c|c|c|}
\hline $\begin{array}{l}\text { Indicadores por município de } \\
\text { residência }\end{array}$ & Cálculo & Fonte/Observação \\
\hline \multicolumn{3}{|l|}{ Chagas } \\
\hline $\begin{array}{l}\text { Cadastros autorreferidos para } \\
\text { doença de Chagas no SIAB }\end{array}$ & $\begin{array}{l}\text { Número de cadastros/população residente * } \\
100.000\end{array}$ & SIAB, dezembro/2015 \\
\hline $\begin{array}{l}\text { Taxa de mortalidade por } \\
\text { doença de Chagas na faixa } \\
\text { etária até } 49 \text { anos }\end{array}$ & $\begin{array}{l}\text { (Número de óbitos < } 50 \text { anos/população residente } \\
\qquad 50 \text { anos) * } 100.000\end{array}$ & $\begin{array}{l}\text { SIM. Média das taxas de } 2014 \text { a } 2016 \\
\text { Registros como causa básica ou associada }\end{array}$ \\
\hline $\begin{array}{l}\text { Taxa de mortalidade por } \\
\text { doença de Chagas na faixa } \\
\text { etária a partir de } 50 \text { anos }\end{array}$ & $\begin{array}{l}\text { (Número de óbitos > } 49 \text { anos/população residente } \\
\qquad>49 \text { anos) * } 100.000\end{array}$ & $\begin{array}{l}\text { SIM. Média das taxas de } 2014 \text { a } 2016 \\
\text { Registros como causa básica ou associada }\end{array}$ \\
\hline \multicolumn{3}{|l|}{ Associadas } \\
\hline $\begin{array}{l}\text { Taxa de internação por } \\
\text { insuficiência cardíaca }\end{array}$ & $\begin{array}{l}\text { (Número de AlH autorizadas/população residente) } \\
\qquad 10.000\end{array}$ & $\begin{array}{l}\text { SIH. Média das taxas de } 2014 \text { a } 2016 \\
\text { Registros com diagnóstico principal - CID-10: I50.0, I50.1 } \\
\text { ou } 150.9\end{array}$ \\
\hline $\begin{array}{l}\text { Taxa de mortalidade por morte } \\
\text { súbita (de origem cardíaca) }\end{array}$ & (Número de óbitos/população residente) * 100.000 & $\begin{array}{l}\text { SIM. Média das taxas de } 2014 \text { a } 2016 \\
\text { Causa básica: CID-10: } 146.1 \text { (retirados registros com } \\
\text { doença de Chagas como secundária) }\end{array}$ \\
\hline \multicolumn{3}{|l|}{ Acesso } \\
\hline $\begin{array}{l}\text { Cobertura populacional } \\
\text { estimada pelas equipes de } \\
\text { atenção básica }\end{array}$ & \multicolumn{2}{|c|}{$\begin{array}{c}\text { Indicador faz parte do rol de Diretrizes, Objetivos, Metas e Indicadores, estabelecidos pela Resolução } \\
\text { no } 5 \text { da Comissão Intergestores Tripartite (http://189.28.128.100/sispacto/SISPACTO_Caderno_Diretrizes_ } \\
\text { Objetivos_2013_2015_3edicao.pdf) }\end{array}$} \\
\hline $\begin{array}{l}\text { Razão de procedimentos } \\
\text { ambulatoriais de média } \\
\text { complexidade relacionados à } \\
\text { doença de Chagas e população } \\
\text { residente }\end{array}$ & $\begin{array}{l}\text { Quantidade aprovada de procedimentos } \\
\text { selecionados de média complexidade, por local de } \\
\text { residência e ano do atendimento (média } 14 \text { a 16)/ } \\
\text { população residente (média } 14 \text { a 16) * } 100\end{array}$ & $\begin{array}{l}\text { SIH (serviços profissionais - procedimento principal ou } \\
\text { secundário) e SIA. Código dos procedimentos CID-10: } \\
\text { 0211020036; 0204030170; 0205010032; 0211020044; } \\
\text { 0204030080; 0204050014; 0212010050; 0303010053; } \\
\text { 0407010270; 0501070028; 0202030776; 0202031047 }\end{array}$ \\
\hline $\begin{array}{l}\text { Razão de procedimentos } \\
\text { ambulatoriais de média } \\
\text { complexidade relacionados à } \\
\text { doença de Chagas e população } \\
\text { residente até } 49 \text { anos }\end{array}$ & $\begin{array}{l}\text { Quantidade aprovada de procedimentos } \\
\text { selecionados de média complexidade até } 49 \text { anos, } \\
\text { por local de residência e ano do atendimento } \\
\text { (média } 14 \text { a 16)/população residente até } 49 \text { anos } \\
\text { (2015) * } 100\end{array}$ & $\begin{array}{l}\text { SIA. Código dos procedimentos CID-10: } 0211020036 ; \\
\text { 0204030170; 0205010032; 0211020044; 0204030080; } \\
\text { 0204050014; 0212010050; } 0501070028 ; 0202030776 ; \\
\text { 0202031047 } \\
\text { SIH. Dados consolidados - código dos procedimentos } \\
\text { CID-10: 0303010053; } 0407010270\end{array}$ \\
\hline $\begin{array}{l}\text { Razão de procedimentos } \\
\text { ambulatoriais de média } \\
\text { complexidade relacionados à } \\
\text { doença de Chagas e população } \\
\text { residente na faixa etária a } \\
\text { partir de } 50 \text { anos }\end{array}$ & $\begin{array}{l}\text { Quantidade aprovada de procedimentos } \\
\text { selecionados de média complexidade }>49 \text { anos, } \\
\text { por local de residência e ano do atendimento } \\
\text { (média } 14 \text { a 16)/população residente }>49 \text { anos } \\
\text { (2015) * } 100\end{array}$ & $\begin{array}{l}\text { SIA. Código dos procedimentos CID-10: } 0211020036 ; \\
\text { 0204030170; 0205010032; } 0211020044 ; 0204030080 ; \\
\text { 0204050014; 0212010050; } 0501070028 ; 0202030776 ; \\
\text { 0202031047 } \\
\text { SIH. Dados consolidados - código dos procedimentos } \\
\text { CID-10: } 0303010053 ; 0407010270\end{array}$ \\
\hline $\begin{array}{l}\text { Proporção de internações por } \\
\text { insuficiência cardíaca com } \\
\text { realização de sorologia IgG } \\
\text { para } T r . \text { cruzi }\end{array}$ & $\begin{array}{c}\text { (Número de AIH com diagnóstico CID-10: I50 } \\
\text { e procedimento secundário } 0202030776 \text { ou } \\
\text { 0202031047)/(Número de AIH com diagnóstico } \\
\text { CID-10: 150) * } 100\end{array}$ & $\begin{array}{l}\text { SIH (serviços profissionais). Média no período de } 2014 \\
\text { a } 2016 \text {. Retiradas as duplicidades para o numerador e } \\
\text { utilizado o incremento frequência para o denominador }\end{array}$ \\
\hline
\end{tabular}

AlH: Autorização de Internação Hospitalar; CID-10: Classificação Internacional de Doenças, 10a revisão; SIA: Sistema de Informações Ambulatoriais; SIAB: Sistema de Informação da Atenção Básica; SIH: Sistema de Internação Hospitalar; SIM: Sistema de Informações sobre Mortalidade.

Nota: População residente 2014 a 2016 (Departamento de Informática do SUS. http://tabnet.datasus.gov.br/cgi/deftohtm.exe?ibge/cnv/poptbr.def, acessado em Jul/2020); população residente por faixa etária - estimativas preliminares elaboradas pelo Coordenação-Geral de Informações e Análises Epidemiológicas, Secretaria de Vigilância e Saúde/Ministério da Saúde, 2015 (Departamento de Informática do SUS.

http://tabnet.datasus.gov.br/cgi/deftohtm.exe?popsvs/cnv/popbr.def, acessado em Jul/2020). 
de soroprevalência da infecção chagásica humana (1975-1980) 18. Essa análise busca avaliar a consistência dos resultados e reduzir a subjetividade no processo de tomada de decisão, identificando qual simulação é mais adequada ao contexto da doença de Chagas crônica.

Ainda buscando validar o modelo, para diminuição da subjetividade, foi realizada análise comparativa utilizando 553 municípios que registraram a ocorrência de triatomíneos com infecção por Tr. cruzi no período de 2014 a 2016, segundo informações repassadas pelas Secretarias Estaduais de Saúde. Além disso, considerando a premissa de que, para cada caso agudo, ocorrem vinte casos silenciosos, foram identificados os percentuais de municípios com registro de casos agudos no Sistema de Informação de Agravos de Notificação (SINAN), no período de 2007 a 2016, que ficaram como média, alta e muito alta prioridade.

O Estado de Goiás incluiu a doença de Chagas crônica na lista estadual de doenças de notificação compulsória em 2013, sendo o único com uma série histórica de notificações da enfermidade. Dessa forma, avaliou-se o percentual de municípios de média, alta e muito alta prioridade de Goiás com mais de uma notificação de doença de Chagas crônica e taxa de deteç̧ão igual a ou maior de 10 casos/100 mil habitantes no período de 2013 a 2018, tendo sido aplicadas as mesmas seis simulações feitas para o Brasil, porém comparando apenas os municípios do estado.

Para a definição da simulação mais adequada, optou-se por aquela que, em sua maioria, teve maior valor de: (i) \% dos municípios com registro de $T$. infestans nos grupos de média, alta e muito alta prioridade; (ii) \% de municípios com prevalência $\geq 4 \%$ no inquérito sorológico e que estão nos grupos de média, alta e muito alta prioridade; (iii) \% de municípios com registro de casos agudos e que estão nos grupos de média, alta e muito alta prioridade; (iv) \% de municípios que registraram a ocorrência de triatomíneos com infecção por Tr. cruzi nos grupos de média, alta e muito alta prioridade; (v) \% dos municípios de Goiás com: média, alta e muito alta prioridade nacional e com mais uma notificação de doença de Chagas crônica e taxa de detecção $\geq 10 / 100$ mil habitantes; registro de $T$. infestans nos grupos de média, alta e muito alta prioridade da simulação estadual; prevalência $\geq 4 \%$ no inquérito sorológico e que estão nos grupos de média, alta e muito alta prioridade na simulação estadual; mais de uma notificação de doença de Chagas crônica e taxa de detecção $\geq 10 / 100$ mil habitantes e que estão nos grupos de média, alta e muito alta prioridade na simulação estadual.

Após a seleção da simulação mais adequada, analisou-se a sensibilidade do modelo proposto, avaliando o impacto de variações nos parâmetros adotados, utilizando o Pradin. Os resultados da simulação também foram analisados segundo as Regiões de Saúde-Comissões Intergestores Regionais (CIR), considerando as medianas dos IMC dos respectivos municípios e a distribuição de alta e muito alta prioridade, além da estratificação realizada para cada estado separadamente, com intuito de subsidiar a tomada de decisão de forma localizada.

\section{Resultados}

A distribuição dos municípios prioritários após a quebra natural do IMC das seis simulações, com respectivos pesos dos índices e indicadores, encontra-se na Figura 1. Considerando o R ${ }^{2}$ da dispersão entre os IMC das simulações, nenhuma das mudanças de indicadores e pesos alterou de forma relevante os resultados, sendo que a quinta simulação apresentou impacto moderado em comparação às demais ( $\mathrm{R}^{2}$ variando de 0,6 a 0,8$)$.

A primeira simulação foi definida como mais adequada por apresentar o maior percentual em sete dos oito critérios de validação propostos, sendo seguida pela quinta em dois critérios (Tabela 1).

Em relação à análise de sensibilidade da primeira simulação, a retirada aleatória de 50 municípios não registrou mudanças significativas nos resultados, demonstrado pela correlação de Pearson entre o IMC original e o calculado na análise de sensibilidade de aproximadamente igual a um, nos três grupos de porte populacional. A retirada das 50 unidades com escores mais baixos e das 50 com escores mais elevados também teve pouco impacto nos resultados da primeira simulação, demonstrando robustez do modelo proposto.

Adotando a primeira simulação, 2.949 (53\%) municípios foram classificados como de média, alta e muito alta prioridade (Tabela 2). As regiões com as maiores proporções de municípios com média, alta e muito alta prioridade foram Sudeste, com $36,11 \%$ dos municípios representados, destacando a 
Figura 1

Distribuição dos municípios brasileiros $(n=5.570)$ prioritários para doença de Chagas crônica nas simulações nacionais com respectivos pesos dos índices e indicadores.

1a) 1a simulação

Prioridade para doença de Chagas crônica (municípios)

Muito baixa (1.275)

Baixa (1.346)

Média (1.345)

Alta (1.003)

Muito alta (601) 1b) 2ª simulação

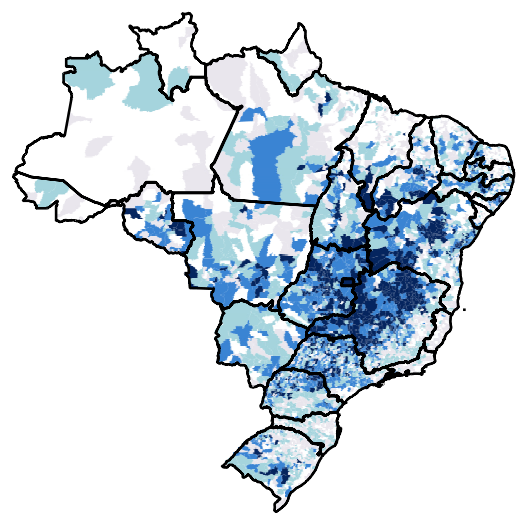
de Chagas crônica (municípios)

$\square$ Muito baixa (1.585)
$\square$ Baixa (1.553)
$\square \quad$ Média (838)
$\square$ Alta (1.027)
$\quad$ Muito alta (567)

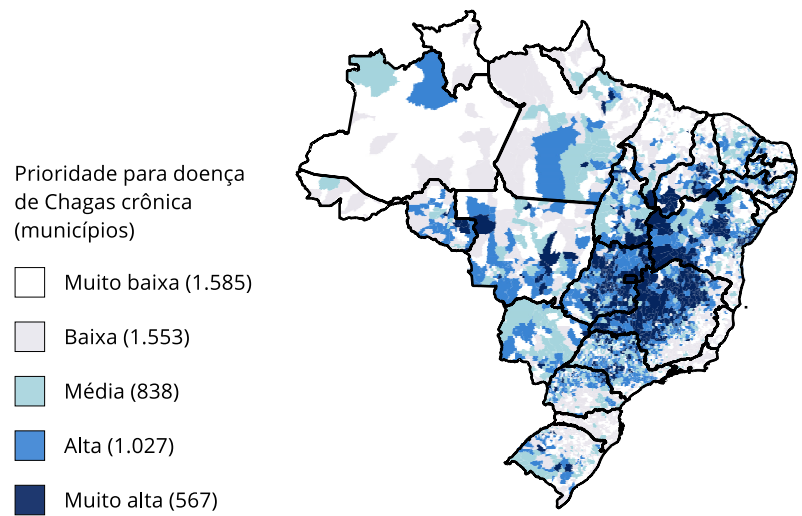

Pesos dos índices:

CHAGAS $=0,5437 ;$ ASSOCIADAS $=0,3460 ;$ ACESSO $=0,1103$

Pesos do indicadores de cada índice:

\begin{tabular}{ll|ll}
\hline Chagas: & SIAB: 1,416 & Acesso: & COB_ABS: 5,299 \\
& SIM_DC $<50: 5,247$ & & RZ_PROC: 0,590 \\
& SIM_DC $>49: 3,338$ & & RZ_PROC $<50: 1,071$ \\
Associadas: & AlH_IC: 5,0 & & RZ_PROC $>49: 1,520$ \\
& MORT_SUB: 5,0 & & \%_EXA_ICC: 1,520 \\
\hline
\end{tabular}

1c) 3ạ simulação

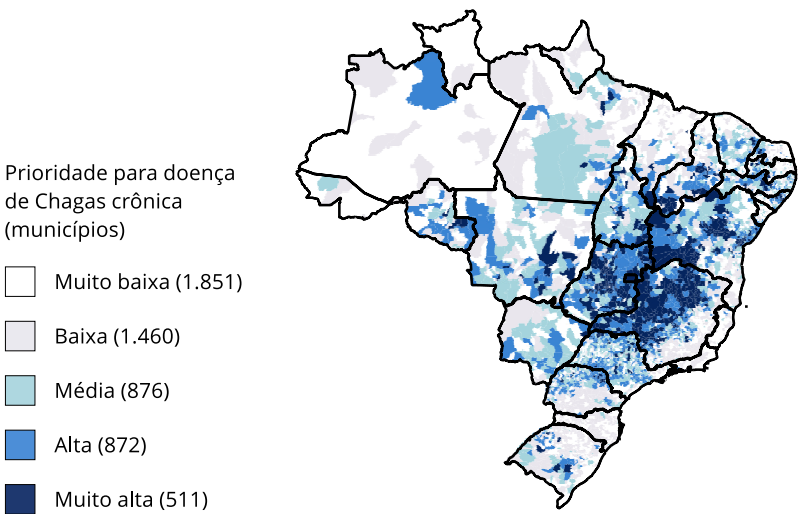

Pesos dos índices:

CHAGAS =0,6551; ASSOCIADAS $=0,2114 ;$ ACESSO $=0,1335$

Pesos do indicadores de cada índice:

\begin{tabular}{ll|ll}
\hline Chagas: & Sem peso & Acesso: & Sem peso \\
\hline Associadas: & Sem peso & & \\
\hline
\end{tabular}

Prioridade para doença

Pesos dos índices:

CHAGAS $=0,6551 ;$ ASSOCIADAS $=0,2114 ;$ ACESSO $=0,1335$

Pesos do indicadores de cada índice:

\begin{tabular}{ll|ll}
\hline Chagas: & SIAB: 1,226 & Acesso: & COB_ABS: 5,617 \\
& SIM_DC $<50: 3,202$ & & RZ_PROC: 1,020 \\
& SIM_DC $>49: 5,571$ & & RZ_PROC $>49: 1,682$ \\
Associadas: & AIH_IC: 5,0 & & \%_EXA_ICC: 1,682 \\
& MORT_SUB: 5,0 & & \\
\hline
\end{tabular}

1d) $4^{a}$ simulação
Prioridade para doença de Chagas crônica (municípios)
Muito baixa (1.927)
Baixa (1.402)
Média (824)
Alta (876)
Muito alta (541)

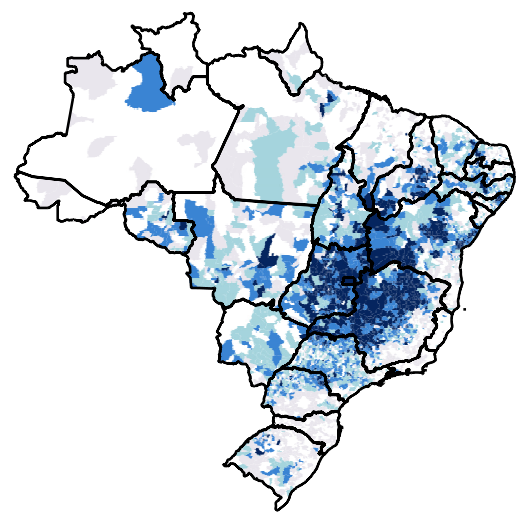

Pesos dos índices:

CHAGAS $=0,7389 ;$ ASSOCIADAS $=0,1602 ;$ ACESSO $=0,1009$ Pesos do indicadores de cada índice:

\begin{tabular}{ll|ll}
\hline Chagas: & Sem peso & Acesso: & Sem peso \\
\hline Associadas: & Sem peso & & \\
\hline
\end{tabular}

(continua) 
Figura 1 (continução)

1e) 5a simulação

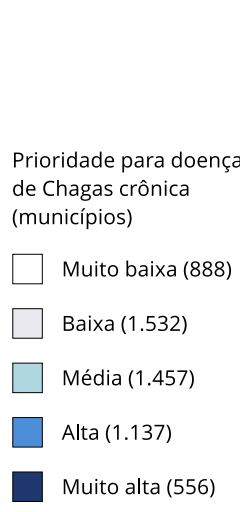

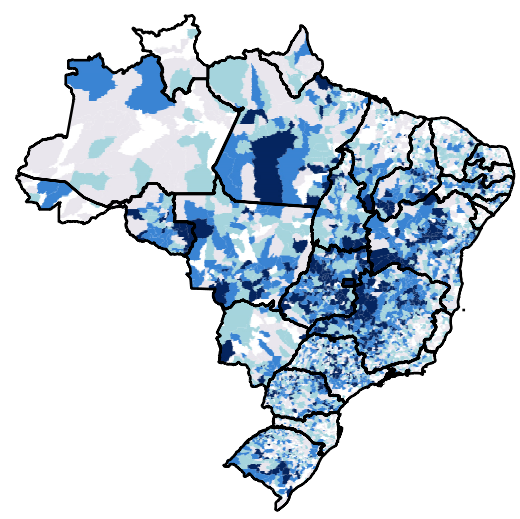

1f) $6^{\underline{a}}$ simulação
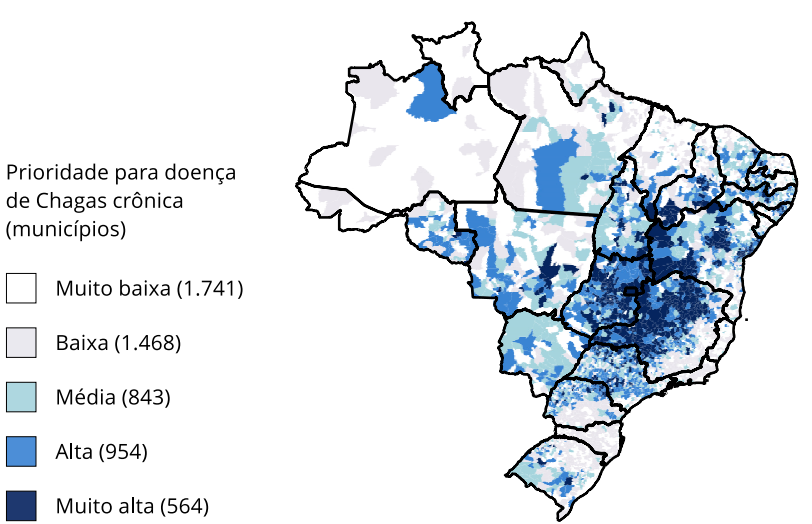

\begin{tabular}{|c|c|c|c|}
\hline \multicolumn{4}{|c|}{$\begin{array}{l}\text { Pesos dos índices: } \\
\text { CHAGAS }=0,333 ; \text { ASSOCIADAS }=0,333 ; \text { ACESSO }=0,333\end{array}$} \\
\hline \multicolumn{4}{|c|}{ Pesos do indicadores de cada índice: } \\
\hline Chagas: & $\begin{array}{l}\text { SIAB: } 1,226 \\
\text { SIM_DC }<50: 3,20 \\
\text { SIM_DC }>49: 5,57\end{array}$ & Acesso & $\begin{array}{l}\text { COB_ABS: } 5,617 \\
\text { RZ_PROC: } 1,020 \\
\text { RZ_PROC }>49: 1,682\end{array}$ \\
\hline Associadas: & $\begin{array}{l}\text { AlH_IC: } 5,0 \\
\text { MORT_SUB: } 5,0\end{array}$ & & $\%$ \%_EXA_ICC: 1,682 \\
\hline
\end{tabular}

\begin{tabular}{|c|c|c|c|}
\hline \multicolumn{4}{|c|}{$\begin{array}{l}\text { Pesos dos índices: } \\
\text { CHAGAS }=0,7071 ; \text { ASSOCIADAS }=0,2014 ; \text { ACESSO }=0,0915\end{array}$} \\
\hline \multicolumn{4}{|c|}{ Pesos do indicadores de cada índice: } \\
\hline Chagas: & $\begin{array}{l}\text { SIAB: } 1,226 \\
\text { SIM_DC }<50: 3,202 \\
\text { SIM_DC }>49: 5,571\end{array}$ & Acesso: & $\begin{array}{l}\text { COB_ABS: } 5,965 \\
\text { RZ_PROC: } 0,986 \\
\text { RZ_PROC }<50: 0,547\end{array}$ \\
\hline Associadas: & $\begin{array}{l}\text { AlH_IC: } 5,0 \\
\text { MORT_SUB: } 5,0\end{array}$ & & $\begin{array}{l}\text { RZ_PROC > 49: } 0,754 \\
\% \text { EXA_ICC: } 1,748\end{array}$ \\
\hline
\end{tabular}

Nota: \%_EXA_ICC: proporção de internações por insuficiência cardíaca com realização de sorologia IgG para Trypanosoma cruzi; AIH_IC: taxa de internação por insuficiência cardíaca; COB_ABS: cobertura populacional estimada pelas equipes de atenção básica; MORT_SUB: taxa de mortalidade por morte súbita (de origem cardíaca); RZ_PROC: razão de procedimentos ambulatoriais de média complexidade; RZ_PROC < 50: razão de procedimentos ambulatoriais de média complexidade e população residente até 49 anos; RZ_PROC > 49: razão de procedimentos ambulatoriais de média complexidade e população residente na faixa etária a partir de 50 anos; SIAB: cadastros autorreferidos para doença de Chagas; SIM_DC < 50: taxa de mortalidade por doença de Chagas na faixa etária até 49 anos; SIM_DC > 49: taxa de mortalidade por doença de Chagas na faixa etária a partir de 50 anos.

contribuição dos municípios dos estados de Minas Gerais e São Paulo, com 74,26\% e 65,42\% respectivamente, seguida das regiões Nordeste, com 26,45\%, e Sul, com 20,18\%. Em menores proporções, estão as regiões Centro-oeste, com 11,87\%, e Norte, com 5,39\% de seus municípios classificados nesses estratos.

Nove capitais foram classificadas como de alta ou muito alta prioridade: Belo Horizonte (Minas Gerais), Brasília, Cuiabá (Mato Grosso), Curitiba (Paraná), Goiânia (Goiás), Maceió (Alagoas), Recife (Pernambuco), Salvador (Bahia) e São Paulo, e cinco com média prioridade: Campo Grande (Mato Grosso do Sul), Fortaleza (Ceará), Palmas (Tocantins), Porto Alegre (Rio Grande do Sul) e Teresina (Piauí). Na Amazônia Legal, estão 87 municípios como de alta e 41 como de muito alta prioridade. Em relação ao porte populacional, dos 601 de muito alta prioridade, três são metrópoles e 24 são de grande, 56 de médio e 518 de pequeno porte.

$\mathrm{Na}$ análise das estratificações estaduais, 3.396 (61\%) municípios foram classificados como de média, alta e muito alta prioridade, um acréscimo de 447 municípios nessa classificação em relação à estratificação nacional (Tabela 2). Esse acréscimo regionalizado ocorreu principalmente no número de municípios das regiões Norte e Nordeste, com aumento de 40,67\% e 23,53\% respectivamente. Em relação aos grupos de prioridade, 2.977 (53\%) municípios permaneceram nos mesmos grupos em ambas as simulações, e $28 \%$ dos classificados nacionalmente como de baixa ou muito baixa prioridade ficaram nos grupos de média, alta e muito alta prioridade estadual. 
Tabela 1

Avaliação das simulações considerando os critérios de validação.

\begin{tabular}{|c|c|c|c|c|c|}
\hline \multirow[t]{3}{*}{ Simulações } & \multicolumn{5}{|c|}{ Critérios de escolha } \\
\hline & $\begin{array}{c}\text { Municípios com } \\
\text { ocorrência de } T \text {. } \\
\text { infestans (1975-1983) } \\
\text { e classificados com } \\
\text { prioridade ME/A/MA }\end{array}$ & $\begin{array}{c}\text { Municípios com } \\
\text { soroprevalência para } \\
\text { doença de Chagas } \\
\geq 4 \%(1975-1980) \text { e } \\
\text { classificados com } \\
\text { prioridade ME/A/MA }\end{array}$ & $\begin{array}{c}\text { Municípios de } \\
\text { Goiás de ME/A/MA } \\
\text { prioridade nacional } \\
\text { com mais de uma } \\
\text { notificação de } \\
\text { doença de Chagas } \\
\text { crônica e taxa de } \\
\text { detecção } \geq 10 / 100 \\
\text { mil habitantes }\end{array}$ & $\begin{array}{l}\text { Municípios com } \\
\text { ocorrência de casos } \\
\text { agudos * de doença } \\
\text { de Chagas (2007- } \\
\text { 2016) e classificados } \\
\text { com prioridade } \\
\text { ME/A/MA }\end{array}$ & $\begin{array}{l}\text { Municípios com } \\
\text { triatomíneos } \\
\text { positivos para } T r . \\
\text { cruzi (2014-2016) e } \\
\text { classificados com } \\
\text { prioridade ME/A/MA }\end{array}$ \\
\hline & $\%$ (n/total) & $\%$ (n/total) & $\%$ (n/total) & $\%($ n/total) & $\%$ (n/total) \\
\hline \multicolumn{6}{|l|}{ Nacional } \\
\hline $1 \underline{a}$ & $85(618 / 729)$ & $77(875 / 1.143)$ & $66(120 / 224)$ & $36(58 / 160)$ & $60(332 / 553)$ \\
\hline $2 \underline{a}$ & $78(569 / 729)$ & $72(823 / 1.143)$ & $53(120 / 226)$ & $34(55 / 160)$ & $58(321 / 553)$ \\
\hline $3 \underline{a}$ & $77(561 / 729)$ & $69(792 / 1.143)$ & $53(120 / 225)$ & $31(49 / 160)$ & $55(304 / 553)$ \\
\hline $4 \underline{a}$ & $76(556 / 729)$ & $69(790 / 1.143)$ & $53(120 / 225)$ & $28(44 / 160)$ & $56(308 / 553)$ \\
\hline $5 \underline{a}$ & $83(607 / 729)$ & $76(869 / 1.143)$ & $66(116 / 218)$ & $51(82 / 160)$ & $59(324 / 553)$ \\
\hline \multirow[t]{3}{*}{$6 \underline{a}$} & $79(575 / 729)$ & $71(816 / 1.143)$ & $53(120 / 227)$ & $31(49 / 160)$ & $57(315 / 553)$ \\
\hline & $\begin{array}{c}\text { Municípios com } \\
\text { ocorrência de } T \text {. } \\
\text { infestans (1975-1983) } \\
\text { e classificados com } \\
\text { prioridade ME/A/MA }\end{array}$ & $\begin{array}{c}\text { Municípios com } \\
\text { soroprevalência para } \\
\text { doença de Chagas } \\
\geq 4 \% \text { (1975-1980) e } \\
\text { classificados com } \\
\text { prioridade ME/A/MA }\end{array}$ & $\begin{array}{c}\text { Municípios de } \\
\text { Goiás com mais de } \\
\text { uma notificação de } \\
\text { doença de Chagas } \\
\text { crônica e taxa de } \\
\text { detecção } \geq 10 / 100 \\
\text { mil casos, com } \\
\text { prioridade ME/A/MA } \\
\% \text { (n/total) }\end{array}$ & & \\
\hline & $\%$ (n/total) & $\%$ (n/total) & $\%($ n/total) & & \\
\hline \multicolumn{6}{|l|}{ Goiás } \\
\hline $1 \underline{a}$ & $74(103 / 139)$ & $81(103 / 127)$ & $94(113 / 120)$ & & \\
\hline $2 \underline{a}$ & $63(88 / 139)$ & $71(90 / 127)$ & $83(99 / 120)$ & & \\
\hline $3 \underline{a}$ & $63(88 / 139)$ & $66(84 / 127)$ & $80(96 / 120)$ & & \\
\hline $4 \underline{a}$ & $63(87 / 139)$ & $66(84 / 127)$ & $82(98 / 120)$ & & \\
\hline $5 \underline{a}$ & $71(98 / 139)$ & $75(95 / 127)$ & $86(103 / 120)$ & & \\
\hline $6 \underline{a}$ & $61(85 / 139)$ & $69(87 / 127)$ & $81(97 / 120)$ & & \\
\hline
\end{tabular}

A: alta; MA: muito alta; ME: média.

* Utilizado município provável de infecção; para os registros em branco ou ignorado, foi considerado município de residência.

Os dados da simulação nacional analisados por CIR indicam 15 delas com todos os municípios classificados com alta ou muito alta prioridade (foram desconsideradas as CIR com apenas um município): dez em Minas Gerais, três em São Paulo e dois em Goiás. Já nas estratificações estaduais, as CIR de alta ou muito alta prioridade estão em Pernambuco, Alagoas, Minas Gerais, São Paulo e Paraná (Figura 2). Encontram-se, entre as CIR de Minas Gerais, as três regiões com maiores medianas dos IMC dos municípios, tanto na simulação nacional quanto nas estratificações estaduais, a saber: Minas Novas/Turmalina/Capelinha; Araçuaí e Francisco Sá. 
Tabela 2

Distribuição dos municípios nas estratificações nacional e estaduais, por região e Unidade da Federação (UF), Brasil.

\begin{tabular}{|c|c|c|c|c|c|c|c|c|c|c|}
\hline \multirow[t]{3}{*}{ Região/UF } & \multicolumn{10}{|c|}{ Número de municípios por prioridade } \\
\hline & \multicolumn{2}{|c|}{ Muito baixa } & \multicolumn{2}{|c|}{ Baixa } & \multicolumn{2}{|c|}{ Média } & \multicolumn{2}{|c|}{ Alta } & \multicolumn{2}{|c|}{ Muito alta } \\
\hline & Nacional & Estadual & Nacional & Estadual & Nacional & Estadual & Nacional & Estadual & Nacional & Estadual \\
\hline Norte & 193 & 76 & 98 & 106 & 83 & 116 & 51 & 89 & 25 & 63 \\
\hline Acre & 17 & 5 & 3 & 5 & 2 & 4 & 0 & 5 & 0 & 3 \\
\hline Amapá & 11 & 2 & 3 & 5 & 2 & 4 & 0 & 4 & 0 & 1 \\
\hline Amazonas & 53 & 16 & 7 & 12 & 2 & 15 & 0 & 9 & 0 & 10 \\
\hline Pará & 62 & 23 & 41 & 25 & 29 & 40 & 8 & 32 & 4 & 24 \\
\hline Rondônia & 11 & 5 & 11 & 15 & 10 & 11 & 14 & 12 & 6 & 9 \\
\hline Roraima & 11 & 3 & 3 & 4 & 1 & 4 & 0 & 3 & 0 & 1 \\
\hline Tocantins & 28 & 22 & 30 & 40 & 37 & 38 & 29 & 24 & 15 & 15 \\
\hline Nordeste & 645 & 358 & 369 & 416 & 382 & 420 & 256 & 345 & 142 & 255 \\
\hline Alagoas & 32 & 27 & 22 & 17 & 21 & 18 & 19 & 24 & 8 & 16 \\
\hline Bahia & 77 & 64 & 57 & 98 & 114 & 103 & 80 & 83 & 89 & 69 \\
\hline Ceará & 74 & 24 & 45 & 51 & 44 & 39 & 20 & 41 & 1 & 29 \\
\hline Maranhão & 112 & 58 & 65 & 58 & 27 & 49 & 8 & 34 & 5 & 18 \\
\hline Paraíba & 95 & 51 & 55 & 49 & 43 & 61 & 20 & 37 & 10 & 25 \\
\hline Pernambuco & 42 & 28 & 29 & 36 & 56 & 45 & 52 & 40 & 6 & 36 \\
\hline Piauí & 61 & 50 & 58 & 50 & 42 & 42 & 42 & 53 & 21 & 29 \\
\hline Rio Grande do Norte & 103 & 40 & 27 & 41 & 24 & 42 & 11 & 20 & 2 & 24 \\
\hline Sergipe & 49 & 16 & 11 & 16 & 11 & 21 & 4 & 13 & 0 & 9 \\
\hline Sudeste & 200 & 253 & 403 & 379 & 395 & 504 & 398 & 329 & 272 & 203 \\
\hline Espirito Santo & 26 & 21 & 43 & 19 & 7 & 24 & 1 & 12 & 1 & 2 \\
\hline Minas Gerais & 83 & 117 & 212 & 166 & 204 & 270 & 171 & 179 & 183 & 121 \\
\hline Rio de Janeiro & 34 & 16 & 39 & 16 & 18 & 32 & 1 & 18 & 0 & 10 \\
\hline São Paulo & 57 & 99 & 109 & 178 & 166 & 178 & 225 & 120 & 88 & 70 \\
\hline Centro-oeste & 60 & 66 & 57 & 90 & 109 & 124 & 147 & 121 & 94 & 66 \\
\hline Distrito Federal & 0 & 0 & 0 & 0 & 0 & 0 & 0 & 0 & 1 & 1 \\
\hline Goiás & 8 & 33 & 14 & 47 & 42 & 62 & 101 & 68 & 81 & 36 \\
\hline Mato Grosso & 35 & 19 & 29 & 30 & 36 & 39 & 29 & 35 & 12 & 18 \\
\hline Mato Grosso do Sul & 17 & 14 & 14 & 13 & 31 & 23 & 17 & 18 & 0 & 11 \\
\hline Sul & 177 & 183 & 419 & 247 & 376 & 314 & 151 & 281 & 68 & 166 \\
\hline Paraná & 18 & 46 & 77 & 73 & 143 & 111 & 105 & 103 & 56 & 66 \\
\hline Rio Grande do Sul & 91 & 97 & 197 & 116 & 152 & 116 & 45 & 93 & 12 & 75 \\
\hline Santa Catarina & 68 & 40 & 145 & 58 & 81 & 87 & 1 & 85 & 0 & 25 \\
\hline Brasil & 1.275 & 936 & 1.346 & 1.238 & 1.345 & 1.478 & 1.003 & 1.165 & 601 & 753 \\
\hline
\end{tabular}


Figura 2

Distribuição das Regiões de Saúde-Comissões Intergestores Regionais (CIR) a partir da mediana do indicador multicriterial (IMC) dos respectivos municípios e percentual de priorização alta (A) e muito alta (MA), nas simulações nacional e estaduais, por macrorregiões brasileiras.

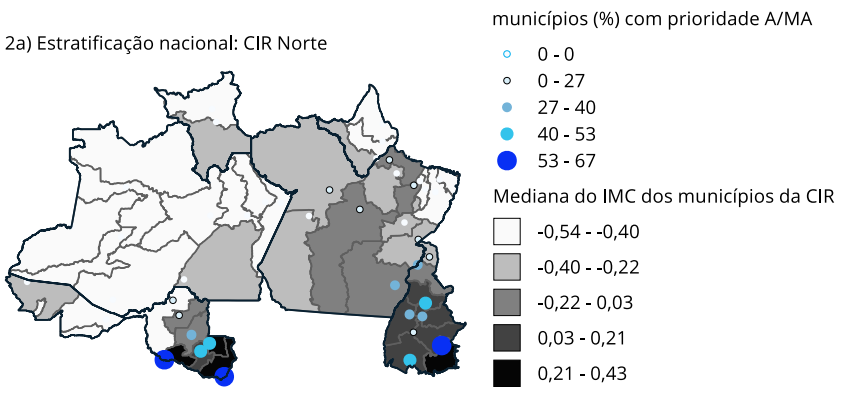

2c) Estratificação nacional: CIR Nordeste

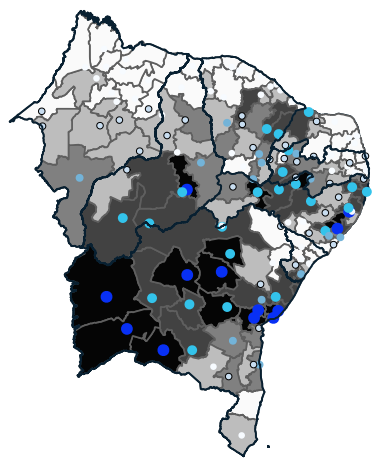

2e) Estratificação nacional: CIR Centro-oeste

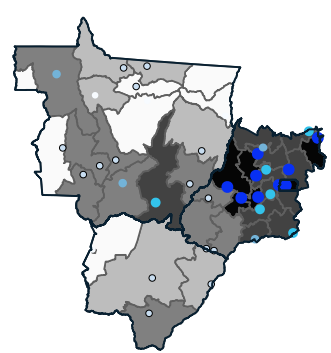

municípios (\%) com prioridade A/MA

- $0,0-0,0$

- $0,0-21,1$

$21,1-33,3$

- $33,3-60,0$

$60,0-88,9$

Mediana do IMC dos municípios da CIR

$-0,61--0,33$

$-0,33--0,15$

$-0,15-0,05$

$0,05-0,26$

$0,26-0,57$ municípios (\%) com prioridade A/MA

- $0-0$

- 0.40

- $40-60$

$60-80$

- $80-100$

Mediana do IMC dos municípios da CIR

$\square-0,33--0,21$

$-0,21-0,00$

$0,00-0,20$

$0,20-0,41$

$0,41-0,67$

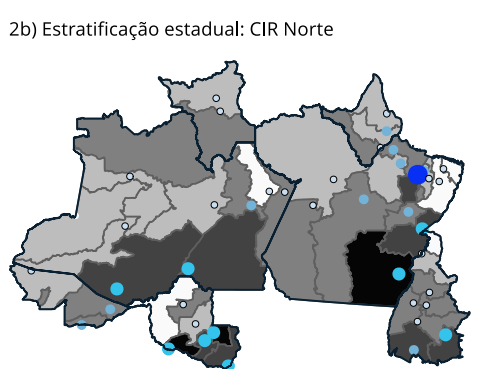

$0-0$

- $0-36$

- $36-53$

$53-71$

- $71-89$

Mediana do IMC dos municípios da CIR $-0,41--0,25$

$-0,25--0,06$

$-0,06-0,09$

$0,09-0,35$

$0,35-0,51$ 2d) Estratificação estadual: CIR Nordeste

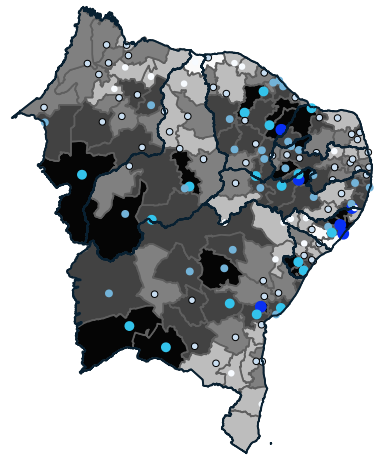

municípios (\%) com prioridade A/MA

$0-0$

$0-40$

- $40-60$

$60-80$

- $80-100$

Mediana do IMC dos municípios da CIR

$-0,70--0,45$

$-0,45--0,23$

$-0,23-0,04$

$0,04-0,30$

$0,30-0,65$

2f) Estratificação estadual: CIR Centro-oeste

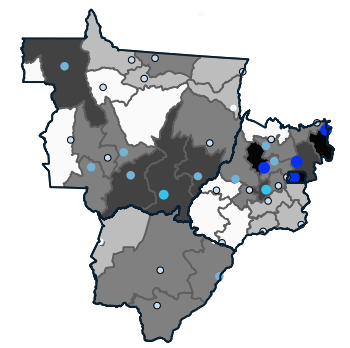

municípios (\%) com prioridade A/MA

$0-0$

- $0-40$

- $40-60$

- $60-80$

$80-100$

Mediana do IMC dos municípios da CIR

$\square-0,42--0,24$

$-0,24--0,05$

$-0,05-0,14$

$0,14-0,30$

$0,30-0,51$

(continua) 


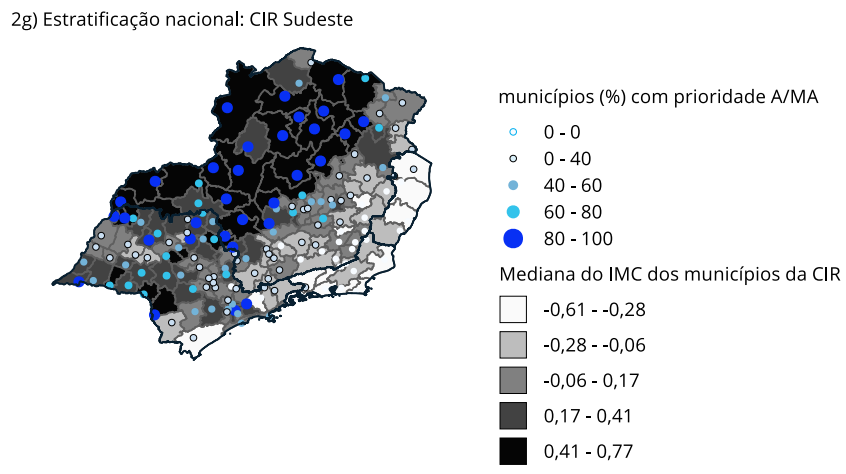

2i) Estratificação nacional: CIR Sul

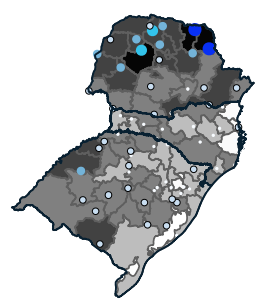

2h) Estratificação estadual: CIR Sudeste

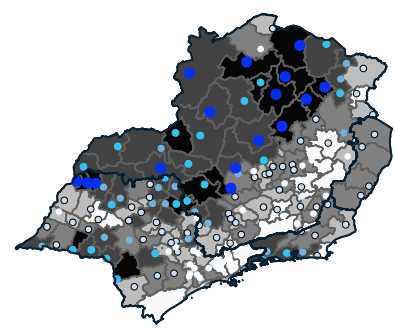

2j) Estratificação estadual: CIR Sul

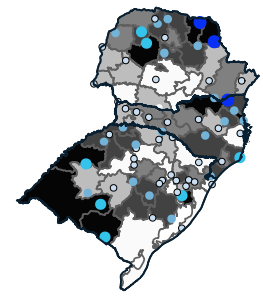

municípios (\%) com prioridade A/MA

$0-0$

$0-40$

- $40-60$

$60-80$

$80-100$

Mediana do IMC dos municípios da CIR

$\square-0,58--0,29$

$-0,29--0,10$

$-0,10-0,09$

$0,09-0,35$

$0,35-0,73$ municípios (\%) com prioridade A/MA

$0-0$

- $0-38$

- $38-57$

- $57-76$

- $76-96$

Mediana do IMC dos municípios da CIR

$\square-0,44--0,33$

$-0,33--0,13$

$-0,13-0,03$

$0,03-0,29$

$0,29-0,53$ municípios (\%) com prioridade A/MA

- $0-0$

- 0.40

- $40-60$

$60-80$

- $80-100$

Mediana do IMC dos municípios da CIR

$\square-0,37--0,22$

$-0,22--0,08$

$-0,08-0,02$

$0,02-0,20$

$0,20-0,53$

\section{Discussão e conclusão}

O modelo selecionado aponta áreas prioritárias para doença de Chagas crônica no âmbito nacional, demonstrando que a AMD é uma ferramenta promissora, considerando as limitações da vigilância em saúde em relação à doença de Chagas, como a não identificação principalmente da fase crônica; a inexistência de sistemas de informação para monitoramento; a fragilidade das informações entomológicas, além da complexidade e diversidade dos serviços de saúde nos territórios brasileiros.

$\mathrm{O}$ uso de informações provenientes de vários níveis deve seguir orientações técnicas objetivas e transparentes, tornando mais específica e confiável a análise, permitindo a elaboração de diagnósticos, a identificação territorial de áreas de intervenção, o monitoramento dos programas e a tomada de decisão 19. Por isso, o modelo utilizou dados epidemiológicos secundários diretos e indiretos, existentes e publicamente disponíveis, para construção de indicadores e identificação no território da presença de indivíduos acometidos pela doença de Chagas crônica.

O acometimento cardíaco na fase crônica da doença é relevante na morbimortalidade, sendo a principal causa de cardiomiopatia não isquêmica na América Latina (países endêmicos), bem como uma das maiores causas de insuficiência cardíaca e morte súbita 20 . Revisão sistemática apontou que a doença de Chagas na América Latina é a etiologia responsável em 1,3\% a 21\% dos casos de insuficiência cardíaca 21 . No Brasil, a insuficiência cardíaca crônica de etiologia chagásica acomete de 4\% a 8\% dos pacientes ambulatoriais e 10\% dos pacientes internados, ao passo que a insuficiência cardíaca descompensada pode ser responsável por até $3 \%$ das internações anuais 22 . A morte súbita é responsável por $55 \%$ a $65 \%$ dos óbitos em pessoas com cardiopatia chagásica crônica ${ }^{23}$. Esses achados justificam o 
uso do sinal insuficiência cardíaca e ocorrência de morte súbita de origem cardíaca como indicadores indiretos da doença de Chagas crônica.

O fato de a simulação mais representativa do território, pelos critérios de validação propostos, não ser aquela com maior peso do índice diretamente relacionado à doença de Chagas crônica, em comparação às demais, reforça a relação dos indicadores indiretos com a doença, sendo apropriados para o modelo proposto.

Entre as simulações realizadas, as alterações de pesos dos índices (Chagas/Associadas/Acesso) não modificaram de forma relevante os resultados, com exceção da quinta simulação, que apresentou a maior proporção de municípios com casos agudos da doença, classificados como média, alta e muito alta prioridade. Isso pode ser explicado pela influência do índice de acesso representando mais o território da Região Norte, visto que os pesos de todos os índices foram iguais, ou seja, neutralizados, tendo todos a mesma relevância.

Para maior variação entre os resultados das simulações, seria necessário mais de uma alteração, como acrescentar indicadores indiretos e alterar significativamente os pesos. Isso se evidencia comparando-se a quarta e a terceira simulações, em que a alteração foi de aproximadamente 10\% do peso dos eixos, resultando em um $\mathrm{R}^{2}$ de 0,99 . Por sua vez, a quarta simulação comparada com a quinta obteve um $\mathrm{R}^{2}$ de 0,56 , pois, além de os índices terem pesos iguais nesta última, houve mudança no peso dos indicadores que compõem cada eixo. Ressalta-se a limitação de usar critério usual como função de preferência em face da falta de parâmetros para doença de Chagas, o que não permite avaliar a magnitude da diferença entre os valores dos índices a partir de limites de indiferença e preferência fraca.

A ausência desses limites pode indicar que alterações temporais, nos valores dos indicadores e respectivos índices, gerem resultados distintos na análise dos critérios de validação em novas simulações, apesar de a análise de sensibilidade demonstrar a robustez da simulação selecionada. Com o intuito de aprimorar o modelo e permitir sua reprodutibilidade, recomenda-se a articulação com stakeholders para definição de limites de indiferença e preferência entre os índices utilizados na priorização.

Estudos populacionais de abrangência nacional foram realizados entre os anos de 1975 a 1980, para determinar a área endêmica para doença de Chagas. Naquele período, as áreas de risco de transmissão da doença de Chagas foram classificadas por meio de dois inquéritos: um sorológico ${ }^{24}$, que não incluiu na amostragem o Distrito Federal e o Estado de São Paulo, apontou para a prevalência da doença acima da média nacional (4,22\%), em áreas concentradas nos municípios pertencentes aos estados do Rio Grande do Sul (8,84\%), Minas Gerais (8,83\%), Goiás (7,4\%), Sergipe (5,97\%) e Bahia (5,44\%); e um estudo entomológico 17 realizado na maioria dos estados brasileiros, à exceção do Estado de São Paulo e dos pertencentes à Região Amazônica, por esta ter sido considerada inicialmente indene. Esses estudos possibilitaram classificar 2.450 municípios de risco para transmissão vetorial em 18 estados, correspondendo a 36\% do território nacional 25.

Comparadas com o conjunto das categorias de priorização - média, alta e muito alta prioridade, há um acréscimo de 9\% (499) dos municípios prioritários para doença de Chagas em relação à área definida na década de 1980. Esse aumento pode ser decorrente da utilização, neste estudo, de dados de toda a população brasileira, enquanto Camargo et al. 24 e Silveira 17 consideraram apenas a área rural do território. Além disso, a doença de Chagas se expandiu das zonas rurais às áreas urbanas e para além das fronteiras dos 21 países da América Latina, onde é considerada endêmica 2,3,4. No Brasil, a migração ocasionou o deslocamento de 500 mil pessoas infectadas por Tr. cruzi para as grandes cidades 26 . Isso pode ser explicado com a classificação de média, alta e muito alta prioridade de 14 capitais, sugerindo a "urbanização da doença”, com a concentração de indivíduos afetados pela doença de Chagas nos grandes centros urbanos. Ou seja, as áreas com maior risco de transmissão vetorial não necessariamente se sobrepõem àquelas com maior prevalência da doença.

Camargo et al. 24 apontaram que 43\% dos municípios brasileiros amostrados tiveram soroprevalência acima de 4,99\%, com maiores proporções de municípios por estado pertencentes às regiões Nordeste (26,44\%), Sul (23,92\%) e Sudeste (23,2\%); em menores proporções, estavam os municípios pertencentes ao Centro-oeste $(17,8 \%)$ e Norte $(8,65 \%)$. Considerando a prevalência maior que a nacional nestas regiões, sugere-se que seus municípios poderiam ser classificados de alto risco para transmissão da doença de Chagas. Adotando essa classificação e comparando com os achados deste estudo, percebe-se que as regiões de média, alta e muito alta prioridade são as mesmas, entretanto há uma inversão na sequência da proporção dos municípios por região, tendo o Sudeste a maior proporção, 
seguido do Nordeste e das demais regiões em menores proporções, o que também pode ser explicado em razão do fluxo migratório principalmente da população do Nordeste do país para o Sudeste.

Vinhaes et al. 27, utilizando também a AMD, com base em indicadores sociodemográficos, econômicos, epidemiológicos e entomológicos para identificar territórios mais vulneráveis à transmissão do Tr. cruzi, excluindo a Região Norte brasileira, encontraram que os municípios mais vulneráveis à transmissão vetorial da doença de Chagas pertenciam principalmente à Região Nordeste. Apesar de não haver dados sistemáticos relativos à prevalência dessa doença, em estudo com revisão sistemática e metanálise publicado em 2014 28, as maiores prevalências regionais foram de 5\% (IC95\%: 3,1-8,1) na Região Nordeste e 5\% (IC95\%: 2,4-9,9) na Região Sudeste, o que também corrobora com os resultados do presente estudo.

A magnitude da Região Nordeste está relacionada principalmente à existência de espécies de triatomíneos com potencial risco para domiciliação em virtude do nicho deixado pelo $T$. infestans principal espécie transmissora da doença de Chagas no Brasil, cuja certificação de eliminação se deu em 2006 29. Dessa forma, é fundamental manter a sensibilidade da vigilância epidemiológica, principalmente para o componente entomológico, fundamental para a interrupção da transmissão e evitar a reemergência da doença onde já está controlada.

As mudanças no perfil da doença de Chagas justificam a inclusão dos municípios do estrato de média prioridade para comparação com as áreas endêmicas apontadas nos inquéritos mencionados, pois, com a transição epidemiológica, esses territórios não necessariamente ficarão com alta e muito alta prioridade, mas espera-se que parte deles esteja classificada com média priorização.

Este estudo apresenta limitações que se devem ao fato de a estratificação nacional não necessariamente refletir o contexto local, já que a metodologia empregada compara todos os municípios entre si. A representatividade da estratificação da região Norte foi reduzida em quase metade da proporção de municípios elencados como prioritários, em comparação com os achados de Camargo et al. 24. Embora seja atualmente a região de maior incidência de casos agudos da doença, foi considerada área indene há 30 anos.

Outra limitação decorre da heterogeneidade do cenário epidemiológico e diversidade dos serviços de saúde. Para minimizar o viés deste último, adotou-se a análise por porte populacional e o acréscimo do índice de acesso, já que municípios com melhor estrutura de serviços de saúde poderiam detectar mais casos.

Por isso, é importante aplicar o modelo para cada Unidade da Federação separadamente e realizar a priorização a partir das regiões de saúde, considerando o dinamismo do território e sabendo que os acometidos pela doença necessitam de cuidado articulado a partir das redes de atenção. Em uma análise multicritério, é importante ter claro o problema e as necessidades para tomada de decisão, pois o processo de estratificação é dinâmico, e a forma como se analisa o território influencia as alternativas para comparação e o resultado final.

Em ambientes reais, o problema decisório tem vários parâmetros e critérios dependentes do juízo de valor e prioridades elencadas pelos diferentes decisores, que podem ser conflitantes entre si 12. Considerando essa complexidade, é útil o emprego de técnicas mais estruturadas de análise, como a AMD. A avaliação multicritério aplicada em vigilância em saúde é um processo dinâmico e depende de cada contexto socioeconômico e epidemiológico, permitindo a análise da localidade e sua influência na produção de fatores de risco nos diferentes locais do território, o que representa um ganho interpretativo, na medida em que cada simulação assume um escore resultante da influência ponderada dos fatores avaliados na localidade.

Considerando que a doença de Chagas é uma enfermidade de difícil detecção, na qual cada caso agudo identificado associa-se a outros 20 casos silenciosos, e que se estima, para o ano de 2020, cerca de 6 milhões de infectados com todas as formas crônicas da doença 4 , torna-se essencial a identificação dos territórios onde essas pessoas estão vivendo. $\mathrm{O}$ envolvimento da população e dos serviços locais de saúde é essencial para manter a sensibilidade do sistema de vigilância. Espera-se que essa estratificação, associada ao monitoramento do risco de transmissão da doença pelas diferentes formas, a exemplo da oral, vetorial e congênita, auxilie a gestão na tomada de decisão e eleição das ações nos territórios prioritários para doença de Chagas, buscando a assistência integral para melhorar a qualidade de vida dos afetados pela enfermidade. 


\section{Colaboradores}

M. M. Lima participou da concepção e delineamento do estudo, coleta, análise e interpretação dos dados, discussão dos resultados, revisão da literatura e redação do manuscrito, além da revisão final do artigo. V. M. Costa colaborou com análise e interpretação dos dados, discussão dos resultados, revisão da literatura e redação do manuscrito e revisão final do artigo. S. L. Palmeira contribuiu na análise e interpretação dos dados, discussão dos resultados e redação do manuscrito e revisão final do artigo. A. P. B. Castro participou da análise e interpretação dos dados, além da revisão final do artigo.

\section{Informações adicionais}

ORCID: Mayara Maia Lima (0000-0002-30128844); Veruska Maia da Costa (0000-0002-68298582); Swamy Lima Palmeira (0000-0001-93892420); André Peres Barbosa de Castro (0000-00024037-6446).

\section{Agradecimentos}

A Rafaella Albuquerque e Silva e Orlando Marcos Faria de Souza, pelas discussões em relação aos aspectos entomológicos da doença de Chagas, e a Liliane da Rocha Siriano (Secretaria de Estado de Saúde de Goiás), por ceder gentilmente os dados anonimizados de casos crônicos de doença de Chagas residentes no Estado de Goiás.

\section{Referências}

1. Chagas C. Nova tripanozomiaze humana: estudos sobre a morfolojia e o ciclo evolutivo do Schizotrypanum cruzi n. gen., n. sp., ajente etiolojico de nova entidade morbida do homem. Mem Inst Oswaldo Cruz 1909; 1:159-218.

2. Lee BY, Bacon KM, Bottazzi ME, Hotez PJ. Global economic burden of Chagas disease: a computational simulation model. Lancet Infect Dis 2013; 13:342-8.

3. Pan American Health Organization. Chagas disease in the Americas: a review of the current public health situation and a vision for the future. Conclusions and recommendations. Washington DC: Pan American Health Organization; 2018.

4. Dias JCP, Ramos AN, Gontijo ED, Luquetti A, Shikanai-Yasuda MA, Coura JR, et al. II Consenso Brasileiro em Doença de Chagas, 2015. Epidemiol Serv Saúde 2016; 25(n. spe):7-86.

5. Nóbrega AA, Araújo WN, Vasconcelos AMN. Mortality due to Chagas disease in Brazil according to a specific cause. Am J Trop Med Hyg 2014; 91:528-33.

6. Ostermayer AL, Passos ADC, Silveira AC, Ferreira AW, Macedo V, Prata AR. O inquérito nacional de soroprevalência de avaliação do controle da doença de Chagas no Brasil (20012008). Rev Soc Bras Med Trop 2011; 44 Suppl II:108-21.

7. Secretaria de Vigilância em Saúde, Ministério da Saúde. Doença de Chagas: 14 de abril - Dia Mundial. Boletim Epidemiológico 2020; 51(n. spe):1-41.

8. Dias JCP. Human Chagas disease and migration in the context of globalization: some particular aspects. J Trop Med 2013; 2013:789758.

9. Ministério da Saúde. Portaria no 1.061 , de 18 de maio de 2020. Revoga a Portaria no 264, de 17 de fevereiro de 2020, e altera a Portaria de Consolidação no 4/GM/MS, de 28 de setembro de 2017, para incluir a doença de Chagas crônica, na Lista Nacional de Notificação Compulsória de doenças, agravos e eventos de saúde pública nos serviços de saúde públicos e privados em todo o território nacional. Diário Oficial da União 2020; 29 mai.

10. Jannuzzi PM, Miranda WL. Pradin e Monit: ferramentas para tomada de decisão no ciclo de políticas públicas. Boletim Estatísticas Públicas 2008; 4:62-73.

11. Longaray AA, Munhoz PRS, Tondolo VAG, Quadro RC. Análise multicritério de decisão e sua aplicação na gestão da saúde: uma proposta de revisão sistemática da literatura. Exacta 2016; 14:609-18.

12. Jannuzzi PM, Miranda WL, Silva DSG. Análise multicritério e tomada de decisão em políticas públicas: aspectos metodológicos, aplicativo operacional e aplicações. Informática Pública 2009; 11:69-87. 
13. Jannuzzi PM. Análise multicritério e a decisão em políticas públicas: implementação da técnica no aplicativo PRADIN e aplicações. In: Escola Nacional de Ciências Estatísticas, organizador. Rio de Janeiro: Escola Nacional de Ciências Estatísticas, Instituto Brasileiro de Geografia e Estatística; 2010. p. 1-35. (Textos para Discussão, 29).

14. Saaty TL. Método de análise hierárquica. Rio de Janeiro: Makron Books do Brasil Editora/ Editora McGraw-Hill; 1991.

15. Gomes LFAM, González MCA, Carignano C. Tomada de decisões em cenários complexos: introdução aos métodos discretos do apoio multicritério à decisão. São Paulo: Pioneira Thomson Learning; 2003.

16. Jenks GF. The data model concept in statistical mapping. International Yearbook of Cartography 1967; 7:186-90.

17. Silveira AC. O inquérito triatomínico (19751983). Rev Soc Bras Med Trop 2011; 44 Suppl II:26-32.

18. Silveira AC, Silva GR, Prata A. O inquérito de soroprevalência da infecção chagásica humana (1975-1980). Rev Soc Bras Med Trop 2011; 44 Suppl II:33-9.

19. Rangel LAD, Gomes LFAM. Multicriteria decision aid in the evaluation of candidates. Production 2010; 20:92-101.

20. Simões MV, Romano MMD, Schmidt A, Martins KSM, Marin-Neto JA. Chagas disease cardiomyopathy. Int J Cardiovasc Sci 2018; 31:173-89.

21. Bocchi EA, Arias A, Verdejo H, Diez M, Gómez E, Castro P. The reality of heart failure in Latin America. J Am Coll Cardiol 2013; 62:949-58.
22. Bocchi EA, Braga FGM, Ferreira SMA, Rohde LEP, Oliveira WA, Almeida DR, et al. III Diretriz Brasileira de Insuficiência Cardíaca Crônica. Arq Bras Cardiol 2009; 93:1-71.

23. Silva VV, Lima AMC, Costa ML, Gomes IP. Perfil dos pacientes chagásicos portadores de cardioversor - desfibrilador implantável (CDI). Int J Cardiovasc Sci 2015; 28:35-41.

24. Camargo ME, Silva GR, Castilho EA, Silveira AC. Inquérito sorológico da prevalência de infecção chagásica no Brasil, 1975/1980. Rev Inst Med Trop São Paulo 1984; 26:192-204.

25. Vinhaes MC, Dias JCP. Doença de Chagas no Brasil. Cad Saúde Pública 2000; 16 Suppl 2:712.

26. Marin-Neto JA, Simões MV, Sarabanda AVL. Chagas' heart disease. Arq Bras Cardiol 1999; 72:264-80.

27. Vinhaes MC, Oliveira SV, Reis PO, Sousa ACL, Silva RA, Obara MT, et al. Assessing the vulnerability of Brazilian municipalities to the vectorial transmission of Trypanosoma cruzi using multi-criteria decision analysis. Acta Trop 2014; 137:105-10.

28. Martins-Melo FR, Ramos AN, Alencar CH, Heukelbach J. Prevalence of Chagas disease in Brazil: a systematic review and meta-analysis. Acta Trop 2014; 130:167-74.

29. Dias JC, Machado EM, Fernandes AL, Vinhaes MC. Esboço geral e perspectivas da doença de Chagas no Nordeste do Brasil. Cad Saúde Pública 2000; 16 Suppl 2:13-34. 


\section{Abstract}

The identification of the magnitude of chronic Chagas disease in Brazil requires linking activities in health surveillance, seeking to develop a wide, hierarchically organized and geographically distributed network of services to provide care to thousands of individuals with Trypanosoma cruzi infection. The study aimed to elaborate a model for prioritization of municipalities for chronic Chagas disease, to offer comprehensive care for persons with the disease. A multicriteria analysis was thus performed using the PROMETHEE II algorithm, implemented in the Pradin software. The criteria for assessing the model consisted of three indices built from the following indicators: (a) epidemiological, directly related to chronic Chagas disease, (b) related to the evolution in chronic Chagas disease, and (c) related to access to health services. Saaty's Fundamental Scale was used to define the indicators' weights, with greater importance assigned to those directly related to chronic Chagas disease and to those with greater reliability and respective quality of information. Assessment of the models' consistency was based on comparison of the available data in historically endemic areas with the distribution of acute cases, besides other sensitivity analyses. The best model was defined by 1,345 municipalities with medium priority, 1,003 high priority, and 601 with very high priority for chronic Chagas disease, with the highest proportions in the Southeast and Northeast regions. Prioritization allows the administration to rationalize and channel resources, and it is essential to identify the territories where persons with chronic Chagas disease are living, to promote comprehensive care and improve quality of life.

Chagas Disease; Decision Support Techniques; Public Health Surveillance

\section{Resumen}

Para el reconocimiento de la magnitud de la enfermedad de Chagas crónica en Brasil, es necesario rearticular las acciones de vigilancia en salud, buscando el desarrollo de una amplia red jerarquizada de servicios distribuida geográficamente, para proveer atención a los millares de ciudadanos afectados por la infección por Trypanosoma cruzi. El objetivo del trabajo fue elaborar un modelo de priorización de municipios para la vigilancia de la enfermedad de Chagas crónica, con el fin de ofertar cuidado integral a las personas afectadas por la enfermedad. Para eso, se realizó un análisis multicriterio, utilizando el algoritmo PROMETHEÉ II, implementado en el software Pradin. Los criterios de evaluación del modelo estuvieron compuestos por tres índices construidos a partir de indicadores (a) epidemiológicos directamente relacionados con la enfermedad de Chagas crónica, (b) derivados de la evolución de la enfermedad de Chagas crónica $y$, (c) relacionados con el acceso a los servicios de salud. La Escala Fundamental de Saaty se utilizó para la definición de los pesos de los indicadores, con mayor importancia a los directamente relacionados con la enfermedad de Chagas crónica $y$ aquellos con mayor confiabilidad, así como su respectiva calidad de información. La evaluación de la consistencia de los modelos se consiguió en comparación con los datos disponibles de las áreas históricamente endémicas, con la distribución de casos agudos, y otros análisis de sensibilidad. El modelo más adecuado se definió por 1.345 municipios de media prioridad, 1.003 de alta y $601 \mathrm{como}$ mucho alta prioridad para enfermedad de Chagas crónica, con mayores proporciones en las regiones Sudeste y Nordeste. La priorización permite a las áreas de gestión racionalizar y dirigir recursos, $y$ es esencial para identificar los territorios donde estas personas están viviendo, con el fin de promover la asistencia integral y mejorar la calidad de vida.

Enfermedad de Chagas; Técnicas de Apoyo para la Decisión; Vigilancia en Salud Pública
Recebido em 06/Jul/2020

Versão final reapresentada em 08/Set/2020

Aprovado em 28/Set/2020 